\title{
Intravitreal Aflibercept in Japanese Patients with Neovascular Glaucoma: The VEGA Randomized Clinical Trial
}

\author{
Masaru Inatani · Tomomi Higashide $\cdot$ Kenji Matsushita • \\ Atsuya Miki · Mari Ueki · Yuji Iwamoto · Masato Kobayashi · \\ Sergio Leal · for the VEGA Investigators
}

Received: October 20, 2020 / Accepted: November 19, 2020 / Published online: December 16, 2020

(C) The Author(s) 2020

\section{ABSTRACT}

Introduction: Neovascular glaucoma is characterized by neovascularization of the iris and the anterior angle chamber. Intravitreal anti-vascular endothelial growth factor agents may

The members list for the VEGA Investigators are mentioned in "Acknowledgements" section.

Electronic supplementary material The online version of this article (https://doi.org/10.1007/s12325020-01579-5) contains supplementary material, which is available to authorized users.

M. Inatani $(\bowtie)$

Department of Ophthalmology, Faculty of Medical Sciences, University of Fukui, Fukui, Japan

e-mail: inatani@u-fukui.ac.jp

T. Higashide

Department of Ophthalmology, Kanazawa

University Graduate School of Medical Science,

Kanazawa, Japan

K. Matsushita · A. Miki

Department of Ophthalmology, Osaka University

Graduate School of Medicine, Osaka, Japan

M. Ueki

Department of Ophthalmology, Osaka Medical

College, Takatsuki-City, Osaka, Japan

Y. Iwamoto · M. Kobayashi

Bayer Yakuhin, Ltd., Osaka, Japan

S. Leal

Bayer Consumer Care AG, Basel, Switzerland improve intraocular pressure (IOP) and neovascularization.

Methods: The VEGA trial assessed the efficacy and safety of intravitreal aflibercept (IVT-AFL) in patients with neovascular glaucoma in a 13-week, randomized, double-masked, shamcontrolled, phase 3 study performed at multiple sites in Japan that enrolled patients with anterior segment neovascularization and IOP $>25 \mathrm{mmHg}$. Patients received background therapy plus IVT-AFL ( $2 \mathrm{mg}$ ) or sham injection at baseline. Patients were re-treated if presenting with $\mathrm{IOP}>21 \mathrm{mmHg}$ and incomplete regression of iris neovascularization, receiving additional sham or IVT-AFL injections at week 1 and IVT-AFL injections at weeks 5 and/or 9. Double-masking was maintained throughout. The primary endpoint was change in IOP from baseline to week 1 .

Results: Fifty-four patients were randomly assigned (full analysis set); the per-protocol set comprised 52 patients. At week 1, the least squares mean change in IOP was $-9.9 \mathrm{mmHg}$ for IVT-AFL versus $-5.0 \mathrm{mmHg}$ for sham [full analysis set: difference $-4.9 \mathrm{mmHg}(95 \%$ confidence interval -10.2 to $0.3 ; P=0.06$ ); perprotocol set: $-5.5 \mathrm{mmHg}(95 \%$ CI -10.8 to $-0.2 ; P=0.04)$ ]. At week 1 , a greater proportion of patients administered IVT-AFL versus sham achieved IOP $\leq 21 \mathrm{mmHg}$ and had improved neovascularization grades. Patients in the sham group who met re-treatment criteria and received IVT-AFL at week 1 [ $n=22(81.5 \%)]$ had 
an additional mean IOP decrease of $9.2 \mathrm{mmHg}$ by week 2 , and the proportion with improvement in neovascularization grades increased from $11.5 \%$ to $69.2 \%$. Increases in the proportion of patients with improved neovascularization grades and the proportion who achieved IOP control ( $\leq 21 \mathrm{mmHg}$ ) were also observed by week 2 in this group. Overall, $77.8 \%$ and $74.1 \%$ of patients treated with IVT-AFL and sham/IVTAFL, respectively, received a single IVT-AFL injection. The most common ocular treatmentemergent adverse event was punctate keratitis (9.3\%: $7.4 \%$ and $11.1 \%$ in the IVT-AFL and sham/IVT-AFL groups, respectively).

Conclusions: IVT-AFL was associated with clinically meaningful improvements in IOP control, indicating that IVT-AFL may be a potential treatment option for patients with neovascular glaucoma.

Trial Registration: Clinicaltrials.gov identifier, NCT02396316.

Keywords: Anti-vascular endothelial growth factor; Anti-VEGF; Intraocular pressure; Intravitreal aflibercept; Neovascular glaucoma; Neovascularization of the angle; Neovascularization of the iris; NVG

\section{Key Summary Points}

Why carry out this study?

Retinal ischemia, thought to be the mediator in most cases of neovascular glaucoma, results in the release of angiogenic factors, such as vascular endothelial growth factor (VEGF), which trigger neovascularization.

It has been suggested that intravitreal antiVEGF agents may improve intraocular pressure (IOP) and neovascularization in patients with neovascular glaucoma.

The VEGA study was designed to assess the efficacy and safety of intravitreal aflibercept (IVT-AFL) in patients with neovascular glaucoma.

\section{What was learned from the study?}

IVT-AFL was associated with clinically meaningful improvements in IOP control (least squares mean difference of - $4.9 \mathrm{mmHg}$ between IVT-AFL and sham) and neovascularization grades, despite not meeting the primary endpoint.

The incidence of treatment-emergent adverse events was consistent with the known safety profile for IVT-AFL.

IVT-AFL may be a potential treatment option for patients with neovascular glaucoma.

\section{DIGITAL FEATURES}

This article is published with digital features, including a summary slide, to facilitate understanding of the article. To view digital features for this article go to https://doi.org/10.6084/ m9.figshare.13251923.

\section{INTRODUCTION}

Neovascular glaucoma (NVG) is characterized by the development of neovascularization of the iris (NVI) and neovascularization of the angle (NVA). The formation of abnormal new blood vessels prevents normal aqueous drainage from the anterior segment of the eye, resulting in elevated intraocular pressure (IOP) $[1,2]$. NVG is reported to account for $0.7-5.1 \%$ of glaucoma cases in the Asian population [3].

Early diagnosis, followed by immediate and aggressive treatment, is needed to prevent complete vision loss in patients with NVG $[2,4]$. Treatment approaches aim to manage both elevated IOP and the underlying cause of the disease [4]. The mainstay therapy for NVG is panretinal photocoagulation (PRP), which, through amelioration of the ischemic retina, leads to shrinkage of the abnormal blood vessels $[2,5]$. However, PRP cannot selectively target pathological tissue, and it damages healthy tissue not involved in neovascularization [6]. 
Given that the regression of neovascularization with PRP is not always immediate, patients may need close monitoring and combined local and systemic treatment to control IOP [6].

Retinal ischemia, thought to be the mediator in the majority of NVG cases [4], results in the release of angiogenic factors, such as vascular endothelial growth factor (VEGF), which trigger neovascularization [2]. It has been suggested that anti-VEGF treatments have a positive influence on outcomes in patients with NVG [7-9]. The results of the VEGA and VENERA studies supported the approval of intravitreal aflibercept (IVT-AFL) injection ( $2 \mathrm{mg}$ ) in 2020 as a treatment for patients with NVG in Japan [10]. Furthermore, clinical guidelines for glaucoma issued by the Japanese Glaucoma Society state that intraoperative or postoperative complications can be suppressed by intravitreal injection of anti-VEGF therapy preoperatively when conducting surgeries for NVG [11].

Here, we report results from the first phase 3 randomized controlled trial (RCT) to assess the short-term efficacy and safety of IVT-AFL compared with sham/IVT-AFL in Japanese patients with NVG treated with IOP-lowering therapy.

\section{METHODS}

\section{Study Design}

VEGA (NCT02396316) was a 13-week, randomized, double-masked, sham-controlled, phase 3 study conducted at 19 study centers in Japan between April 2, 2015, and September 6, 2016, to evaluate the efficacy and safety of IVT-AFL in patients with NVG. The study was conducted in accordance with the Declaration of Helsinki and the International Council for Harmonization guideline E6: Good Clinical Practice. Institutional review board approval of the protocol was obtained at each site (see supplementary material), and all patients provided written informed consent.

Patients were randomly assigned $1: 1$ to receive either IVT-AFL $2 \mathrm{mg}$ or a sham injection on day 1 (baseline), and double-masking was maintained during the study (eFigure 1). Randomization was stratified by baseline in stage of
NVG (open- or closed-angle) and was generated by Bayer Pharma AG's Randomization Management through an interactive voice response system and interactive web response system.

Additional treatment was administered only if re-treatment criteria (IOP $>21 \mathrm{mmHg}$, incomplete regression of iris neovascularization, and IVT-AFL was deemed necessary by the investigator), as evaluated by the masked investigator, were met. A combination of $\geq 3$ topical IOP-lowering drugs was administered during a run-in phase before the first treatment and was kept unchanged until the pre-injection IOP evaluation at week 1 . PRP was performed on and after day 1 as needed. Systemic IOP-lowering drugs were prohibited for $24 \mathrm{~h}$ before the pre-injection IOP evaluation at baseline and week 1. Patients were evaluated for the development of NVI and NVA by gonioscopy in conjunction with slit-lamp microscopy. NVI and NVA were assessed in the study eye using the NVI and NVA grading systems on a scale of 0 to 4 (Table 1) [12]. IOP was assessed using applanation tonometry [Goldmann (standard technique) or Tono-Pen (Reichert)]. Noncontact tonometry was not permitted (see online supplement for further details).

\section{Patients}

Japanese patients aged $\geq 20$ years were eligible for inclusion if they had a diagnosis of NVG with neovascularization in the anterior segment (iris and anterior chamber angle) and IOP $>25 \mathrm{mmHg}$ due to neovascularization in the study eye. Key exclusion criteria included angle closure from conditions other than NVG, use of topical ophthalmic atropine sulfate hydrate in the study eye $\leq 30$ days before day 1 , and use of systemic IOP-lowering drugs $24 \mathrm{~h}$ before the pre-injection IOP evaluation on day 1. Full exclusion criteria are provided in the online supplement.

\section{Endpoints}

The primary endpoint was the mean change in IOP from baseline to week 1 (pre-injection IOP evaluation). The secondary endpoint was the 
Table 1 Grading systems for neovascularization of the iris and neovascularization of the angle [12]

\begin{tabular}{|c|c|c|}
\hline Grade & Neovascularization of iris & Neovascularization of angle \\
\hline 0 & No iris neovascularization & No angle neovascularization \\
\hline 1 & $\begin{array}{l}\text { Fine surface neovascularization of the pupillary zone of } \\
\text { the iris involving less than } 2 \text { quadrants }\end{array}$ & $\begin{array}{l}\text { Fine neovascular twigs crossing the scleral spur and } \\
\text { ramifying on the trabecular meshwork involving less } \\
\text { than } 2 \text { quadrants }\end{array}$ \\
\hline 2 & $\begin{array}{l}\text { Surface neovascularization of the pupillary zone of the } \\
\text { iris involving more than } 2 \text { quadrants }\end{array}$ & $\begin{array}{l}\text { Neovascular twigs crossing the scleral spur and } \\
\text { ramifying on the trabecular meshwork involving more } \\
\text { than } 2 \text { quadrants }\end{array}$ \\
\hline 3 & $\begin{array}{l}\text { In addition to neovascularization of the pupillary zone, } \\
\text { neovascularization of the ciliary zone of the iris and/ } \\
\text { or ectropion uveae involving } 1-3 \text { quadrants }\end{array}$ & $\begin{array}{l}\text { In addition to neovascularization of the trabecular } \\
\text { meshwork, peripheral anterior synechiae (PAS) } \\
\text { involving } 1-3 \text { quadrants }\end{array}$ \\
\hline 4 & $\begin{array}{l}\text { In addition to neovascularization of the pupillary zone, } \\
\text { neovascularization of the ciliary zone of the iris and/ } \\
\text { or ectropion uveae involving more than } 3 \text { quadrants }\end{array}$ & $\begin{array}{l}\text { In addition to neovascularization of the trabecular } \\
\text { meshwork, PAS involving more than } 3 \text { quadrants }\end{array}$ \\
\hline
\end{tabular}

proportion of patients with a change of $\geq 1$ NVI grade from baseline to week 1. Exploratory endpoints included the proportion of patients with a change of $\geq 1$ NVA grade from baseline to week 1 and the proportion of patients who achieved IOP $\leq 21 \mathrm{mmHg}$ at week 1 . Additional efficacy endpoints were evaluated at weeks 2,5 , 9 , and 13 . Safety was evaluated throughout the study.

\section{Statistical Analysis}

A sample size of 27 patients in each group had $90 \%$ power to detect a difference in means using a $t$ test with a 2 -sided significance level of 0.05 under the assumption that the mean \pm standard deviation (SD) of the decrease in IOP from baseline to predose at week 1 was $10 \pm 12 \mathrm{mmHg}$ for the IVT-AFL group and $0 \pm 10 \mathrm{mmHg}$ for the sham group. For the primary endpoint, the difference in the changes between the treatment groups (IVT-AFL minus sham) and their 2-sided 95\% confidence interval (CI) were estimated by using an analysis of covariance model, including treatment group and stage of NVG (open or closed angle) at baseline (as randomly assigned) as the fixed effect and baseline IOP as a covariate. Analysis of the primary endpoint was conducted on the full analysis set (FAS). The superiority of IVTAFL over sham was established if the upper limit of the 2-sided 95\% CI for the difference (IVTAFL minus sham) was less than 0. For patients with no predose IOP at week 1, the missing value was imputed by the last post-baseline, predose IOP assessment before week 1 [last observation carried forward (LOCF)]. The sensitivity analysis was conducted in the per-protocol set (PPS), which included all patients in the FAS who had available predose IOP measurements at both baseline and week 1 and no major protocol violations (i.e., wherein the violation might have affected assessment of efficacy). For the secondary endpoint, the proportions of patients who had improved NVI grade from baseline were summarized descriptively. The point estimate of the treatment difference (IVT-AFL minus sham) at week 1 and its 2-sided 95\% CI stratified by stage of NVG (as randomly assigned) using Mantel-Haenszel weights were estimated, with LOCF for patients with no NVI grade at week 1. All additional efficacy variables were summarized descriptively. For the proportions, the point estimates 
of the treatment difference (IVT-AFL group minus sham group) at week 1 and their 2-sided 95\% CIs stratified by stage of NVG using Mantel-Haenszel weights were also estimated. Statistical analysis was performed with the software package SAS v.9.2 or higher (SAS Institute, Cary, NC, USA).

\section{RESULTS}

\section{Patients}

In total, 63 patients were enrolled and 54 were randomly assigned (FAS). The PPS included 52 patients (26 patients in each group); 2 patients were excluded, due to accidental unmasking and missing inclusion criteria. All patients completed week 1 (primary endpoint), while 42 patients completed treatment (Fig. 1). All the patients were Japanese, most were male $(83.3 \%)$, and the mean age was 67.1 years (Table 2). At baseline, approximately twice as many patients in the IVT-AFL group had retinal vein occlusion, and the prevalence of grade 3 or 4 NVI or NVA was approximately $25 \%$ higher, whereas baseline IOP was higher in the sham/ IVT-AFL group.

The use of systemic IOP-lowering drugs was numerically greater in the sham/IVT-AFL group compared with the IVT-AFL group [59.3\% (16/ 27 patients) and $40.7 \%$ (11/27 patients), respectively]. Overall, of the 27 patients who received systemic IOP-lowering drugs, all received acetazolamide; 2 patients also received mannitol and 1 received glycerol.

\section{Ocular Surgery in Study Eye}

Three patients ( 1 in the IVT-AFL group, 2 in the sham group) previously underwent surgery (retinal laser coagulation) in the study eye. After the start of study treatment, 26 patients (14 in the IVT-AFL group, 12 in the sham/IVT-AFL group) had ocular surgery. The most common concomitant surgery performed for the treatment of NVG was laser PRP (11 in the IVT-AFL group, 10 in the sham/IVT-AFL group), and the second most common was trabeculectomy ( 2 in the IVT-AFL group, 1 in the sham/IVT-AFL group).

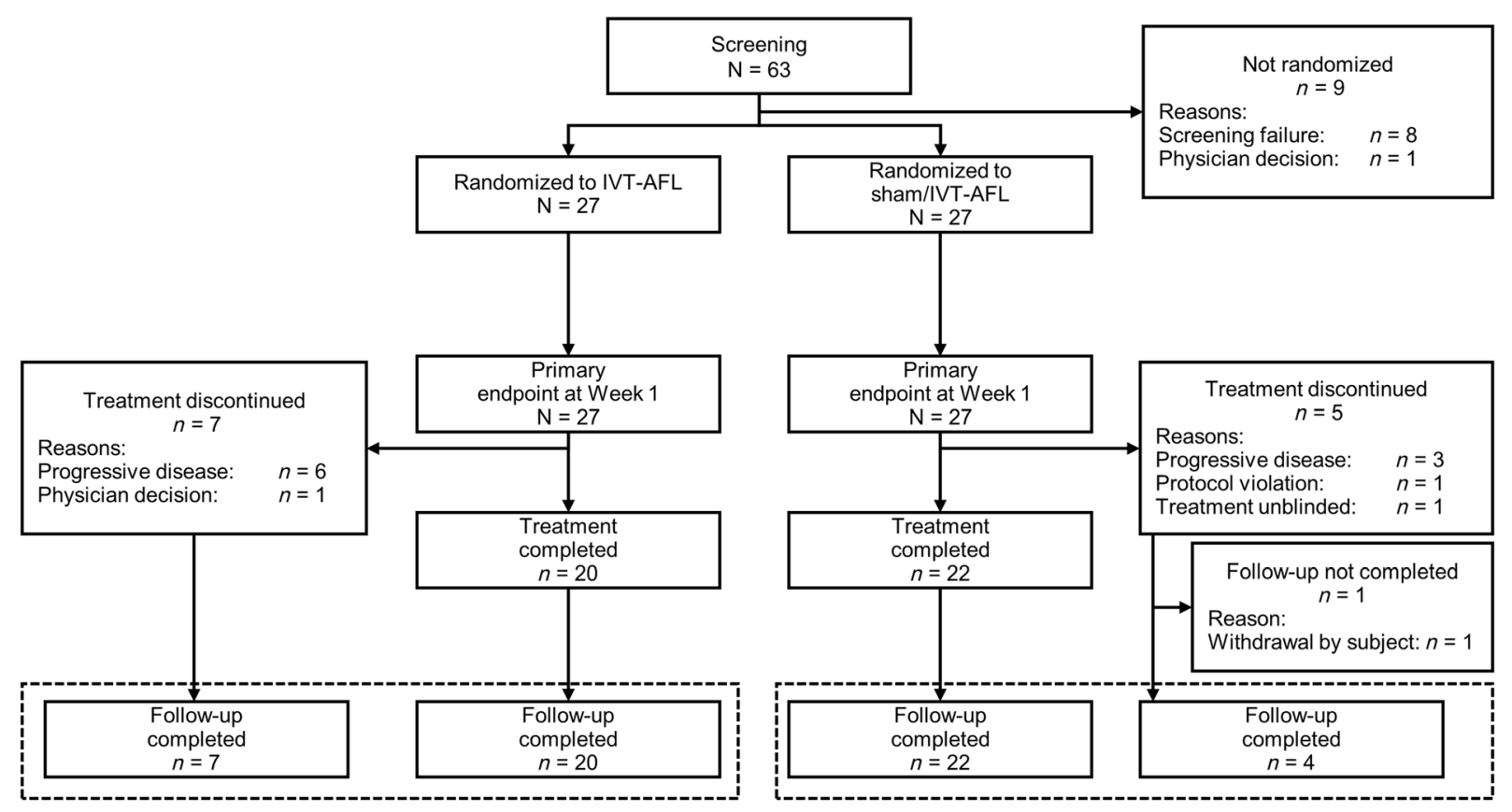

Fig. 1 Patient flowchart; $I V T$-AFL intravitreal aflibercept 
Table 2 Baseline demographics and disease characteristics

\begin{tabular}{llll}
\hline & $\begin{array}{l}\text { IVT-AFL 2 } \mathbf{~ m g} \\
\mathbf{n}=\mathbf{2 7}\end{array}$ & $\begin{array}{l}\text { Sham/IVT-AFL } \\
\boldsymbol{n}=\mathbf{2 7}\end{array}$ & $\begin{array}{l}\text { Total } \\
\boldsymbol{n}=\mathbf{5 4}\end{array}$ \\
\hline Male, $n$ (\%) & $22(82)$ & $23(85)$ & $45(83)$ \\
Mean age, years (SD) & $68.1(13)$ & $66.2(14)$ & $67.1(13)$ \\
Primary diagnosis, $n(\%)$ & & & $23(43)$ \\
Proliferative diabetic retinopathy & $10(37)$ & $13(48)$ & $16(30)$ \\
Central retinal vein occlusion & $11(41)$ & $5(19)$ & $9(17)$ \\
Ocular ischemic syndrome & $4(15)$ & $5(19)$ & $6(11)$ \\
Other & $2(7)$ & $4(15)$ & $27(50)$ \\
Duration of disease, $\leq 6.0$ days, $n$ (\%) & $17(63)$ & $10(37)$ & $47(87)$ \\
Stage of NVG, $n$ (\%) & & $23(85)$ & $7(13)$ \\
Open angle & $24(89)$ & $4(15)$ & $20(37)$ \\
Closed angle & $3(11)$ & $7(26)$ & $34(63)$ \\
NVI grade 3 or 4 at baseline (pooled), $n(\%)$ & $13(48)$ & $15(56)$ & $35(10)$ \\
NVA grade 3 or 4 at baseline (pooled), $n(\%)$ & $19(70)$ & $37(9)$ & \\
Mean IOP at baseline, mmHg (SD) & $33(10)$ &
\end{tabular}

IOP intraocular pressure, $I V T-A F L$ intravitreal aflibercept, $N V A$ neovascularization of the angle, $N V G$ neovascular glaucoma, $N V I$ neovascularization of the iris, $S D$ standard deviation

\section{Treatment}

All 27 patients in the IVT-AFL group received treatment on day 1 , and most patients $(n=22)$ in the sham/IVT-AFL group received IVT-AFL at week 1. Only $4 / 27$ patients had the sham injection and did not require an IVT-AFL injection at weeks 1,5 , or 9; PRP was received by $2 / 4$ patients, and trabeculectomy was received by 1 patient. Overall, $77.8 \%$ and $74.1 \%$ of patients in the IVT-AFL group and the sham/ IVT-AFL group, respectively, received only 1 IVT-AFL injection (eTable 1). The mean (SD) number of IVT-AFL injections over the study period was $1.2(0.4)$ in the IVT-AFL group and $1.0(0.6)$ in the sham/IVT-AFL group.

\section{Efficacy}

The mean change in IOP from baseline to week 1 was numerically greater in the IVT-AFL group
$(-8.5 \mathrm{mmHg})$ than in the sham/IVT-AFL group $(-4.9 \mathrm{mmHg})$. The primary endpoint, least squares mean change in IOP from baseline to week 1 , was $-9.9 \mathrm{mmHg}$ in the IVT-AFL group and $-5.0 \mathrm{mmHg}$ in the sham/IVT-AFL group (FAS, LOCF); the mean difference was not statistically significant $\quad(-4.9 \mathrm{mmHg} ; \quad 95 \% \quad$ CI -10.2 to $0.3 ; P=0.06$ ), and the primary endpoint was not met. For the sensitivity analysis, based on the PPS, the least squares mean difference in favor of IVT-AFL was $-5.5 \mathrm{mmHg}$ (95\% CI -10.8 to $-0.2 ; P=0.04$ ) (Fig. 2a).

At week $1,81.5 \%$ of patients randomized to sham met re-treatment criteria and received an IVT-AFL injection. This group had a substantial additional reduction in IOP between week 1 and week 2 (- $9.2 \mathrm{mmHg})$. In both groups, IOP levels remained stable after week 2 until the end of the study, with minimal need for re-injection (Fig. 2b). At week 5, 4 patients (14.8\%) in each group met re-treatment criteria and received an additional IVT-AFL injection. At week 9, 2 
patients $(7.4 \%)$ in the IVT-AFL group and 1 patient $(3.7 \%)$ in the sham/IVT-AFL group were re-treated.

A greater proportion of patients in the IVTAFL group had improved NVI grades (by $\geq 1$ grade) at week 1 (pre-injection) than in the sham/IVT-AFL group $[70.4 \%$ vs. $11.5 \%$,
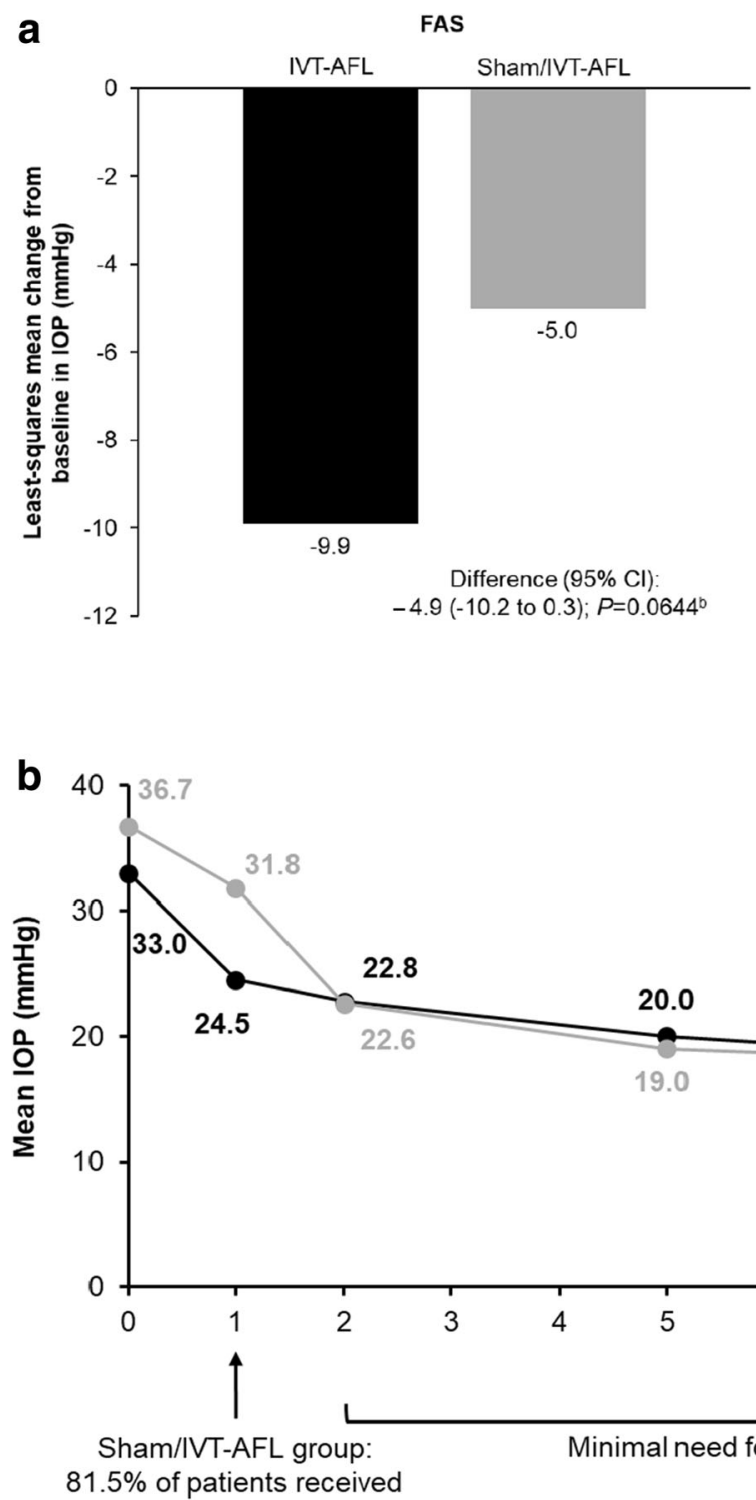

IVT-AFL

Fig. 2 Mean change in IOP from baseline to week 1 (a) and mean IOP from baseline to week 13 (b). ${ }^{a} \mathrm{~A}$ sensitivity analysis of the primary endpoint was performed on the PPS. ${ }^{\mathrm{b}}$ Calculated using ANCOVA model, with treatment group and stage of NVG randomization as fixed effects, with baseline value as covariate; respectively; Mantel-Haenszel adjusted difference $59.1 \%$ (95\% CI 37.0-81.2)] for the FAS (LOCF) (Fig. 3a). At week 1, 80.8\% and $7.7 \%$ of patients in the sham/IVT-AFL group had stable or worsened NVI grades, respectively, compared with $29.6 \%$ and $0.0 \%$ in the IVT-AFL group, respectively. From week 1 to week 2 , the

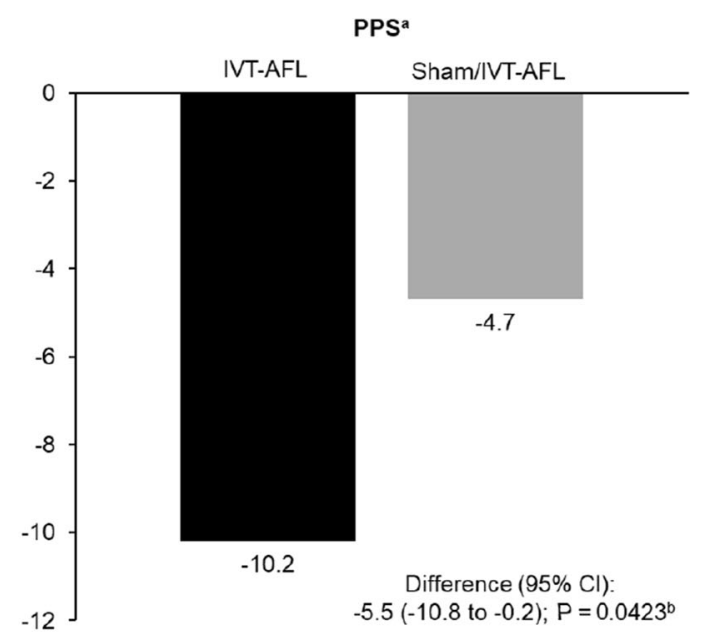

IVT-AFL $\quad-\bullet-$ Sham/IVT-AFL

ANCOVA analysis of covariance, $F A S$ full analysis set, IOP intraocular pressure, $I V T-A F L$ intravitreal aflibercept, $N V G$ neovascular glaucoma, $P P S$ per-protocol set 
a

IVT-AFL $-\odot$ Sham/IVT-AFL

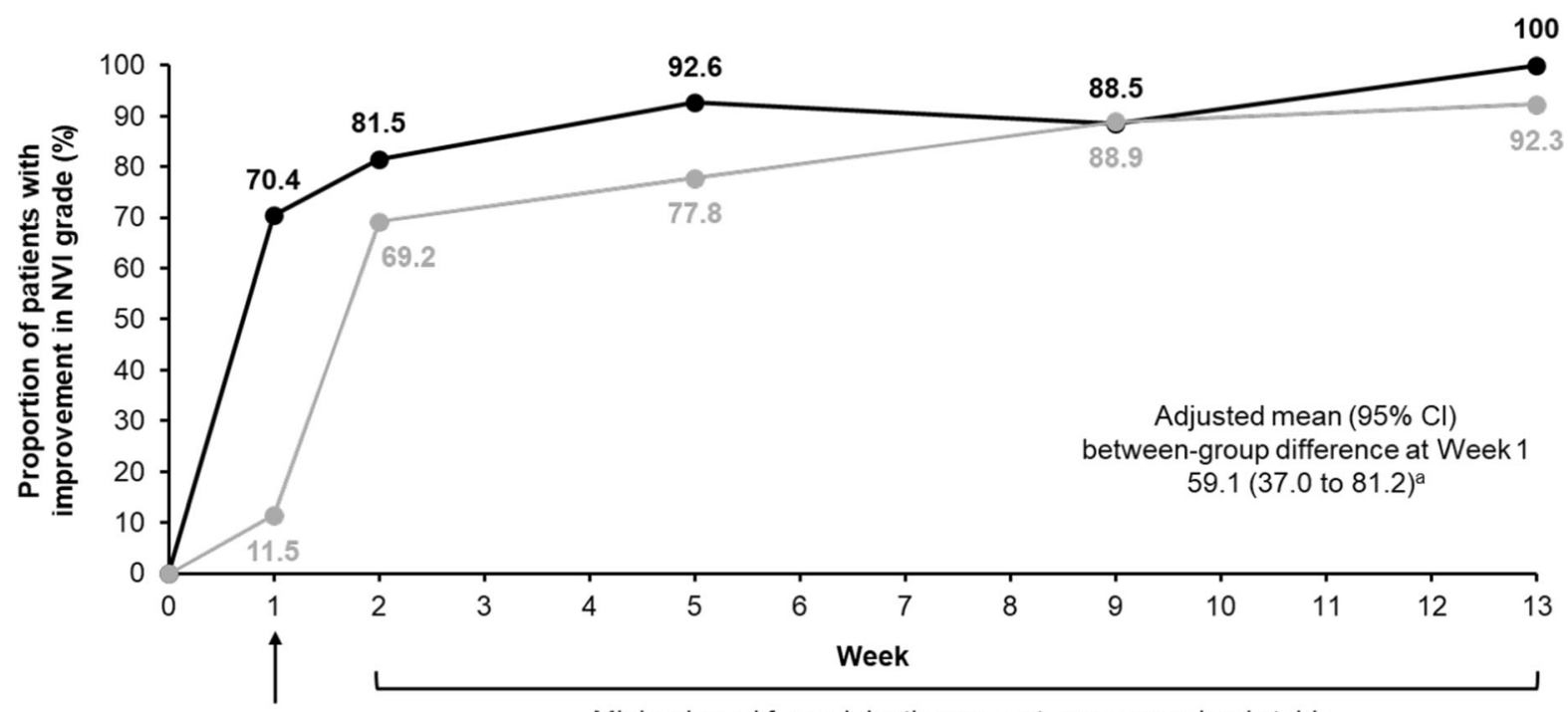

Sham/IVT-AFL group:

Minimal need for re-injections as outcomes remained stable

$81.5 \%$ of patients received

IVT-AFL

b

-IVT-AFL $\quad-$-Sham/IVT-AFL

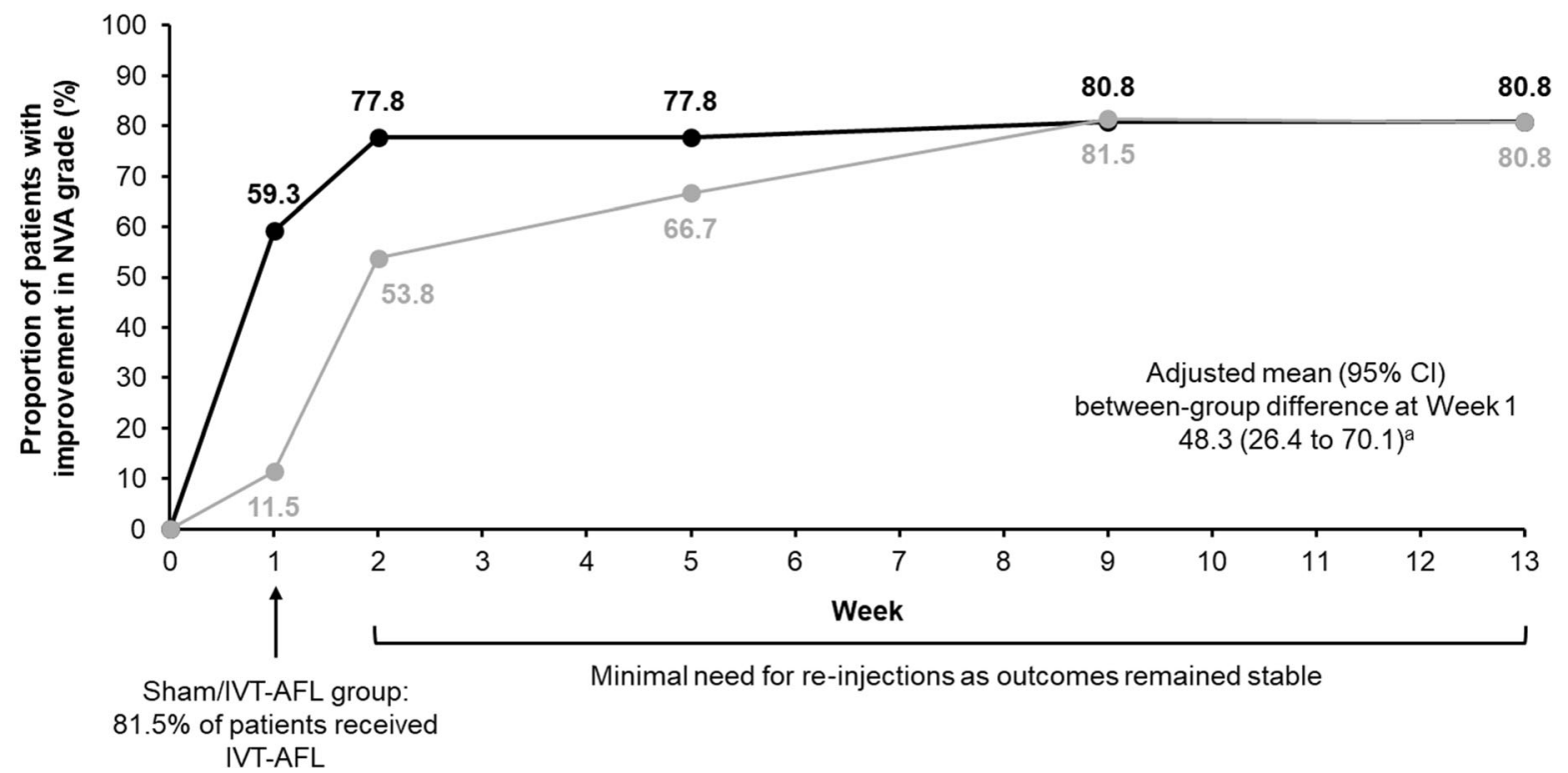

Fig. 3 Improvements ( $\geq 1$ grade) in NVI (a) and NVA (b) from baseline to week $13{ }^{a}$ Calculated using Mantel-Haenszel weights adjusted for stage of NVG for randomization (FAS, LOCF). FAS full analysis set,
$I V T$-AFL intravitreal aflibercept, $L O C F$ last observation carried forward, $N V A$ neovascularization of the angle, $N V G$ neovascular glaucoma, $N V I$ neovascularization of the iris 
Table 3 TEAEs at week 13

\begin{tabular}{|c|c|c|c|}
\hline No. (\%) of patients with AEs & $\begin{array}{l}\text { IVT-AFL } \\
n=27\end{array}$ & $\begin{array}{l}\text { Sham/IVT-AFL } \\
n=27\end{array}$ & $\begin{array}{l}\text { Total } \\
n=54\end{array}$ \\
\hline Any TEAE & $13(48.1)$ & $20(74.1)$ & $33(61.1)$ \\
\hline Ocular TEAEs & $11(40.7)$ & $15(55.6)$ & $26(48.1)$ \\
\hline \multicolumn{4}{|c|}{ Commonly reported ocular TEAEs ( $\geq 5 \%$ overall) } \\
\hline Punctate keratitis & $2(7.4)$ & $3(11.1)$ & $5(9.3)$ \\
\hline Eye pain & 0 & $3(11.1)$ & $3(5.6)$ \\
\hline Conjunctival hemorrhage & $2(7.4)$ & $1(3.7)$ & $3(5.6)$ \\
\hline Injection-site pain & $2(7.4)$ & $1(3.7)$ & $3(5.6)$ \\
\hline Procedural pain & $3(11.1)$ & 0 & $3(5.6)$ \\
\hline Nonocular TEAEs & $8(29.6)$ & $11(40.7)$ & $19(35.2)$ \\
\hline \multicolumn{4}{|c|}{ Commonly reported nonocular TEAEs ( $\geq 5 \%$ overall) } \\
\hline Constipation & $1(3.7)$ & $2(7.4)$ & $3(5.6)$ \\
\hline Headache & $1(3.7)$ & $2(7.4)$ & $3(5.6)$ \\
\hline Treatment-related TEAEs & $1(3.7)$ & $2(7.4)$ & $3(5.6)$ \\
\hline SAEs & $3(11.1)$ & $3(11.1)$ & $6(11.1)$ \\
\hline Ocular SAEs in study eye & $2(7.4)$ & $1(3.7)$ & $3(5.6)$ \\
\hline Nonocular SAEs & $1(3.7)$ & $2(7.4)$ & $3(5.6)$ \\
\hline Treatment-related SAEs & 0 & $1(3.7)^{\mathrm{a}}$ & $1(1.9)$ \\
\hline APTC-AEs & 0 & $1(3.7)^{\mathrm{a}}$ & $1(1.9)$ \\
\hline
\end{tabular}

$A E$ adverse event, $A P T C$ Antiplatelet Trialists' Collaboration, $A T E$ arterial thromboembolic event, $I V T-A F L$ intravitreal aflibercept, $S A E$ serious adverse event, TEAE treatment-emergent adverse event

a This APTC-defined ATE was nonfatal myocardial infarction, considered by investigator to be drug related. Patient was randomly assigned to the sham group, and IVT-AFL was given at week 1

proportions of patients with improved NVI grade in the study eye increased from $11.5 \%$ to $69.2 \%$ in the sham/IVT-AFL group compared with $70.4 \%$ to $81.5 \%$ in the IVT-AFL group.

Similarly, for the change in NVA grades from baseline to week 1 , a greater proportion of patients in the IVT-AFL group (59.3\%) had improved NVA grades (by $\geq 1$ grade) at week 1 (pre-injection) than in the sham/IVT-AFL group [11.5\%; Mantel-Haenszel adjusted difference 48.3\% (95\% CI 26.4-70.1)] (Fig. 3b). In addition, at week $1,76.9 \%$ and $11.5 \%$ of patients in the sham/IVT-AFL group had stable or worsened
NVA grades, respectively, compared with $40.7 \%$ and $0.0 \%$ in the IVT-AFL group, respectively. From week 1 to week 2 , the proportion of patients with improved NVA grade in the study eye increased from 11.5 to $53.8 \%$ in the sham/ IVT-AFL group compared with $59.3 \%$ to $77.8 \%$ in the IVT-AFL group. In both treatment groups, improvements were maintained to week 13 .

The proportion of patients who achieved well-controlled IOP $(\leq 21 \mathrm{mmHg})$ at week 1 (pre-injection) was numerically higher in the IVT-AFL group (44.4\%) than in the sham/IVTAFL group (7.4\%), and it increased to $59.3 \%$ at 
week 2 in the IVT-AFL group (eFigure 2). However, in the sham/IVT-AFL group, the proportion of patients who achieved IOP $\leq 21 \mathrm{mmHg}$ increased to $63.0 \%$ at week 2 after $81.5 \%$ of sham-treated patients received IVT-AFL at week 1. In both treatment groups, controlled IOP was maintained to week 13.

The results of subgroup analysis should be interpreted with caution due to the small number of patients; however, results indicated that none of the patient demographics or disease characteristics [e.g., male/female sex or primary diagnosis leading to development of NVG (retinal vein occlusion, proliferative diabetic retinopathy)] evaluated affected the efficacy of IVT-AFL in terms of improvement in IOP, NVI, and NVA grades (data not shown).

\section{Safety}

Treatment-emergent adverse events (TEAEs) during the study period were reported in $48.1 \%$ and $74.1 \%$ of the IVT-AFL and sham/IVT-AFL groups, respectively (Table 3). Among them, $3.7 \%$ and $7.4 \%$ of patients in the IVT-AFL and sham/IVT-AFL groups, respectively, reported study drug-related TEAEs. Treatment-emergent serious adverse events were reported by $11.1 \%$ of patients in both groups. Most adverse events were mild or moderate but there were three severe ocular TEAEs that were due to underlying ocular disease (IVT-AFL group: retinal vein occlusion and retinal artery occlusion; sham/ IVT-AFL group: diabetic retinopathy). Up to week 13, Antiplatelet Trialists' Collaborationdefined arterial thrombo-embolic events were reported in 1 patient (3.7\%) in the sham/IVTAFL group (nonfatal myocardial infarction after IVT-AFL injection at week 1).

\section{DISCUSSION}

This phase 3 RCT evaluating the efficacy and safety of IVT-AFL in NVG is the first study of its kind in Japanese patients. Although the primary endpoint was missed, treatment with IVT-AFL resulted in clinically meaningful numerical reductions in IOP, together with regression of NVI and NVA, in higher proportions than those observed with sham/IVT-AFL treatment. After a single IVT-AFL injection, there was a mean reduction in IOP from baseline of $8.5 \mathrm{mmHg}$ compared with $4.9 \mathrm{mmHg}$ in the sham/IVT-AFL group. The primary analysis showed that the reduction in IOP in the IVT-AFL group was in line with the estimated change $(-10 \pm 12 \mathrm{mmHg})$ used for determination of the sample size; however, the sham/IVT-AFL group experienced a larger reduction than the expected $0 \pm 10 \mathrm{mmHg}$. Thus, the difference did not achieve statistical significance in the primary analysis, but the prespecified sensitivity analysis showed a statistically significant difference: the least squares mean difference was $-5.5 \mathrm{mmHg} \quad(95 \% \quad \mathrm{CI}-10.8$ to -0.2 ; $P=0.04)$. Overall, the narrowly missed primary endpoint in the context of sizable reductions in IOP in the sham/IVT-AFL group leaves open the hypothesis that a benefit for patients who received IVT-AFL might have passed undetected, a hypothesis that requires further research.

Overall, at week 1, outcome measures of treatment success were greater in the IVT-AFL group than the sham/IVT-AFL group, including change from baseline in IOP, proportion of patients with improved NVI grade, change from baseline in NVI and NVA, and proportion of patients who achieved IOP control ( $\leq 21 \mathrm{mmHg}$ ). Additionally, the latter outcomes improved in the sham/IVT-AFL group from week 1 to week 2 following administration of IVT-AFL to patients who met the re-treatment criteria at week 1 , further suggesting a beneficial effect of IVT-AFL in this patient population. Overall, most patients (75.9\%) required only 1 IVT-AFL injection. These findings suggest that IVT-AFL treatment may lead to a clinically meaningful and sustained reduction in IOP, which is essential to prevent damage to the optic nerve and subsequent vision loss in patients with NVG $[1,5]$. Only a minority of patients $(22.2 \%)$ required more than one injection of IVT-AFL, and, although the number of patients in subgroups were small, the efficacy of IVT-AFL was not affected by the patient demographic factors or disease characteristics evaluated. Studies have shown variation in the duration of complete VEGF suppression 
following intravitreal anti-VEGF injections, which may account for some of the differences in efficacy observed [13]. IVT-AFL treatment also resulted in regression of NVI and NVA, preventing development of fibrovascular membrane on the anterior surface of the iris and the iridocorneal angle of anterior chamber, thereby reducing the formation of anterior synechiae and angle closure [1, 14]. This ultimately prevents mechanical blockage of the aqueous humor outflow, resulting in increased IOP [2].

While the need for RCTs investigating the impact of anti-VEGF therapy in patients with NVG has been highlighted [2], most reports are case studies with small numbers of patients and inconclusive results. A prospective case series of IVT-AFL in patients with newly diagnosed NVG demonstrated rapid regression of NVI and NVA, with stabilization or reduction of IOP at 52 weeks [9]. The efficacy of intravitreal ranibizumab in patients with NVG has only been demonstrated in case studies, which have shown rapid and sustained IOP-lowering effects when given in addition to conventional therapy. One case series of 10 patients with NVG found that adjuvant treatment with ranibizumab led to a rapid and significant reduction in mean IOP from baseline to day 14 that was sustained at the 12-month follow-up [8]. Another case series, involving 18 patients with IOP $\geq 25 \mathrm{mmHg}$ despite treatment with the maximum medical therapy, demonstrated controlled IOP with 2.7 injections of ranibizumab without additional surgery in 10 patients [7]. The present study provides evidence, in an RCT setting, of a potential beneficial effect of IVTAFL for lowering IOP and regression of NVA and NVI. Safety results of the VEGA study were consistent with the established safety profile of IVT-AFL.

In patients with NVG, anti-VEGF drugs are expected to rapidly decrease IOP and subsequently produce an early relief of high IOP and symptoms. Previous data reporting practical experience with anti-VEGF agents, as well as general considerations about the disease, suggest that an early endpoint is appropriate to detect whether the treatment with anti-VEGF is able to provide earlier control of the disease manifestations than standard treatment options. The evaluation timing for the primary endpoint was therefore determined to be at week 1 [7-9].

Limitations of this study include that, for ethical reasons, treatment with systemic IOPlowering treatments was permitted throughout the study, because elevated IOP can quickly lead to irreversible loss of vision [2]. The use of topical IOP-lowering drugs was mandatory, and systemic treatments were permitted, except within $24 \mathrm{~h}$ before baseline and at week 1 visits. Imbalances in the frequency of these treatments across both arms might have influenced the outcome. Analysis of potential confounding factors established that use of systemic IOPlowering drugs emerged as the most likely association for the higher than expected reduction in IOP observed in the sham/IVT-AFL group. In the sham/IVT-AFL group, mean IOP reductions of clinically meaningful magnitude (>5 mmHg) were observed only in the subgroup of patients who received systemic IOPlowering drugs; in the IVT-AFL group, both subgroups showed similar reductions (eTable 2). This also demonstrates that IOP after the run-in phase remained stable despite continued application of topical IOP-lowering drugs, and that the maximum effect had been achieved. In both treatment groups, patients with higher baseline IOP received systemic IOP-lowering drugs more frequently than those with lower baseline IOP; and almost all patients with a baseline IOP of $\geq 40 \mathrm{mmHg}$ (all except 1 patient in the IVTAFL group) received systemic IOP-lowering drugs. In the IVT-AFL group, a clinically meaningful reduction in IOP was observed in patients with all ranges of baseline IOP, whereas in the sham/IVT-AFL group, a clinically meaningful reduction in IOP was observed only in patients with a baseline IOP $\geq 40 \mathrm{mmHg}$ (eTable 3 ). Since the reduction in IOP from baseline to week 1 in the sham/IVT-AFL group was observed mainly in patients with high baseline IOP, it is likely that this clinically meaningful reduction may have been due to the influence of systemic IOP-lowering drugs. Diabetic retinopathy is associated with reduced kidney function [15]. Thus, the 24-h period when use of IOP-lowering treatments was not permitted might have been insufficient to ensure washout. 
A further hypothesis could be that the increase in IOP after renal clearance of the drug might have been slower due to ischemia of the ciliary processes in patients with high IOP. We should also note that the study did not control for diurnal variation in IOP. As a result of the findings from this study, the VENERA study was designed to exclude the influence of systemic IOP-lowering drugs and PRP on IOP [16].

\section{CONCLUSION}

In conclusion, this first phase 3 study indicates a potentially positive efficacy and safety profile for IVT-AFL in Japanese patients with NVG. IVTAFL (on a background of IOP-lowering therapy and PRP) was associated with clinically meaningful improvements in IOP control and regression of anterior segment neovascularization compared with sham/IVT-AFL treatment. Furthermore, the safety outcomes during the study were consistent with the known safety profile of IVT-AFL.

\section{ACKNOWLEDGEMENTS}

The authors thank all the patients and investigators who contributed to this study. The members list for the VEGA Investigators: Tomomi Higashide, Masaru Inatani, Chie Sotozono, Shigeru Kinoshita, Kenji Matsushita, Mari Ueki, Kanji Takahashi, Toshiaki Kubota, Takashi Koto, Yasuyuki Takai, Shinichiro Teranishi, Katsuyoshi Suzuki, Toru Nakazawa, Tomoyasu Shiraya, Tomohiro Oshiro, Hitoshi Takagi, Daisuke Nagasato, Hideyasu Oh.

Funding. Funding for the study, medical writing and editorial assistance for this manuscript, and funding for the Rapid Service Fee and Open Access Fee was provided by Bayer Consumer Care AG, Basel, Switzerland. In conjunction with the VEGA steering committee, Bayer participated in the design of the study; analysis and interpretation of the data; preparation, review, and approval of the manuscript; and decision to submit the manuscript for publication. Additionally, Bayer was responsible for the conduct of the study and oversight of the collection and management of data.

Medical Writing and Editorial Assistance. Medical writing and editorial support for the preparation of this manuscript (under the guidance of the authors) was provided by Louise Brady, PhD, of ApotheCom (UK), funded by Bayer Consumer Care AG, Basel, Switzerland.

Authorship. All named authors meet the International Committee of Medical Journal Editors (ICMJE) criteria for authorship for this article, take responsibility for the integrity of the work as a whole, and have given their approval for this version to be published.

Authorship Contributions. All authors contributed to the design; data acquisition, analysis, and interpretation; and preparation and final review of the manuscript. All authors approved the manuscript for submission.

Prior Presentation. The results of the VEGA study have been presented in part at EURETINA 2020 Virtual, 2-4 October, 2020.

Disclosures. Dr Masaru Inatani reports nonfinancial support from Bayer during the conduct of the study and personal fees from Bayer outside the submitted work. Dr Tomomi Higashide reports receiving personal fees from Bayer. Dr Kenji Matsushita reports receiving travel fees from Bayer Yakuhin and Senju and receiving lecture fees from Alcon, Alcon Pharmaceutical, Kowa, Nitten, Otsuka, Pfizer, Santen, Senju, Maruho, and Bayer Yakuhin. Dr Atsuya Miki reports receiving personal fees from Topcon, Otsuka Pharmaceuticals, Kowa Pharmaceuticals, Ellex Inc., Pfizer Japan, Santen Pharmaceutical, Alcon Pharmaceuticals, Hoya Corporation, Senju Pharmaceutical, JFC Sales Plan, Sucampo Pharmaceuticals, Sensimed, and Seed and grants from JSPS Kakenhi and the Charitable Trust Fund for Ophthalmic Research in Commemoration of Santen Pharmaceutical's Founder. Dr Mari Ueki reports no conflicts of interest. Mr Yuji Iwamoto and $\mathrm{Dr}$ Masato Kobayashi are employees of Bayer Yakuhin, 
Ltd., Osaka, Japan. Dr Sergio Leal is an employee of Bayer Consumer Care AG, Basel, Switzerland, and reports a patent pending (WO2018/229,034).

Compliance with Ethics Guidelines. The study was conducted in accordance with the Declaration of Helsinki and the International Council for Harmonization guideline E6: Good Clinical Practice. Institutional review board approval of the protocol was obtained at each site (see supplementary material), and all patients provided written informed consent.

Data Availability. Availability of the data underlying this publication will be determined according to Bayer's commitment to the EFPIA/ PhRMA "Principles for responsible clinical trial data sharing." This pertains to scope, time point, and process of data access. As such, Bayer commits to sharing, upon request from qualified scientific and medical researchers, patientlevel clinical trial data, study-level clinical trial data, and protocols from clinical trials in patients for medicines and indications approved in the United States (US) and European Union (EU) as necessary for conducting legitimate research. This applies to data on new medicines and indications that have been approved by the EU and US regulatory agencies on or after January 1, 2014. Interested researchers can use www. clinicalstudydatarequest.com to request access to anonymized patient-level data and supporting documents from clinical studies to conduct further research that can help advance medical science or improve patient care. Information on the Bayer criteria for listing studies and other relevant information is provided in the Study sponsors section of the portal. Data access will be granted to anonymized patient-level data, protocols, and clinical study reports after approval by an independent scientific review panel. Bayer is not involved in the decisions made by the independent review panel. Bayer will take all necessary measures to ensure that patient privacy is safeguarded.

Open Access. This article is licensed under a Creative Commons Attribution-
NonCommercial 4.0 International License, which permits any non-commercial use, sharing, adaptation, distribution and reproduction in any medium or format, as long as you give appropriate credit to the original author(s) and the source, provide a link to the Creative Commons licence, and indicate if changes were made. The images or other third party material in this article are included in the article's Creative Commons licence, unless indicated otherwise in a credit line to the material. If material is not included in the article's Creative Commons licence and your intended use is not permitted by statutory regulation or exceeds the permitted use, you will need to obtain permission directly from the copyright holder. To view a copy of this licence, visit http:// creativecommons.org/licenses/by-nc/4.0/.

\section{REFERENCES}

1. Olmos LC, Lee RK. Medical and surgical treatment of neovascular glaucoma. Int Ophthalmol Clin. 2011;51(3):27-36.

2. Simha A, Braganza A, Abraham L, Samuel P, Lindsley K. Anti-vascular endothelial growth factor for neovascular glaucoma. Cochrane Database Syst Rev. 2013;10(10):CD007920.

3. Liao N, Li C, Jiang H, Fang A, Zhou S, Wang Q. Neovascular glaucoma: a retrospective review from a tertiary center in China. BMC Ophthalmol. 2016;16:14.

4. Havens SJ, Gulati V. Neovascular glaucoma. Dev Ophthalmol. 2016;55:196-204.

5. Rodrigues GB, Abe RY, Zangalli C, et al. Neovascular glaucoma: a review. Int J Retina Vitreous. 2016;2: 26.

6. Andres-Guerrero V, Perucho-Gonzalez L, GarciaFeijoo J, et al. Current perspectives on the use of anti-VEGF drugs as adjuvant therapy in glaucoma. Adv Ther. 2017;34(2):378-95.

7. Shen X, Chen Y, Wang Y, Yang L, Zhong Y. Intravitreal ranibizumab injection as an adjuvant in the treatment of neovascular glaucoma accompanied by vitreous hemorrhage after diabetic vitrectomy. J Ophthalmol. 2016;2016:4108490. 
8. Luke J, Nassar K, Luke M, Grisanti S. Ranibizumab as adjuvant in the treatment of rubeosis iridis and neovascular glaucoma-results from a prospective interventional case series. Graefes Arch Clin Exp Ophthalmol. 2013;251(10):2403-13.

9. SooHoo JR, Seibold LK, Pantcheva MB, Kahook MY. Aflibercept for the treatment of neovascular glaucoma. Clin Exp Ophthalmol. 2015;43(9):803-7.

10. Pharmaceuticals and Medical Devices Agency. March 25, 2020; https://www.info.pmda.go.jp/go/ pack/1319405A1027_1_12/?view=frame\&style= XML\&lang=ja.

11. Tanihara $H$, Aihara $M$, Inatani M, Inoue $T$, Kiuchi $Y$. The Japan glaucoma society guidelines for glaucoma. Nippon Ganka Gakkai Zasshi. 2018;122: 5-53.
12. Teich SA, Walsh JB. A grading system for iris neovascularization prognostic implications for treatment. Ophthalmology. 1981;88(11):1102-6.

13. Fauser S, Schwabecker V, Muether PS. Suppression of intraocular vascular endothelial growth factor during aflibercept treatment of age-related macular degeneration. Am J Ophthalmol. 2014;158(3): 532-6.

14. Hayreh SS. Neovascular glaucoma. Progr Retinal Eye Res. 2007;26(5):470-85.

15. Zhang H, Wang J, Ying GS, Shen L, Zhang Z. Diabetic retinopathy and renal function in Chinese type 2 diabetic patients. Int Urol Nephrol. 2014;46(7):1375-81.

16. Inatani M, Higashide $T$, Matsushita $K$, et al. Efficacy and safety of intravitreal aflibercept injection in Japanese patients with neovascular glaucoma: outcomes from the VENERA Study. Adv Ther. 2020. 